\title{
Estudio de la influencia del Periodismo de Prevención en la comunicación institucional. Llamada de atención a la Administración
}

institucional.us.es/ambitos/

\author{
Silvia Alende Castro \\ Universidad de Vigo \\ silvia.alende@gmail.com \\ Mercedes Román Portas \\ Universidad de Vigo \\ mroman@uvigo.es
}

English Version: Study of Prevention Journalism effects on institutional communication. Warning Call to the Public Administration.

\begin{abstract}
Resumen
A través de una actitud consciente por parte de los periodistas y los medios, el Periodismo de Prevención busca aproximarse a la cotidianeidad del lector para la gestión de riesgos en el entorno próximo. En este contexto, se estudia cómo esta perspectiva -en concreto su fase denominada Llamada de atención a la Administración- influye en la comunicación de las instituciones. Metodológicamente, se revisaron más de 30.000 unidades informativas en 406 ejemplares de diarios españoles. A través del análisis de contenido y del análisis estadístico multivariante

HJ Biplot, se han testado datos de presencia y temática de este tipo de mensaje
\end{abstract}

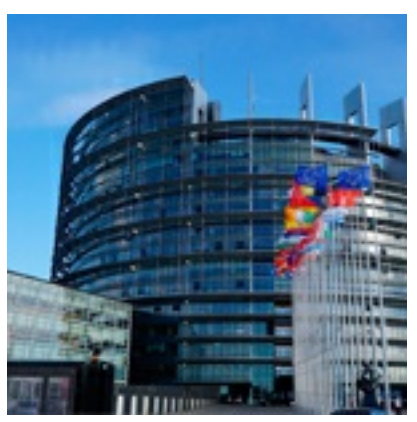
periodístico.

\section{Palabras clave}

Comunicación, comunicación institucional, periodismo, Periodismo de Prevención.

\begin{abstract}
Prevention Jounalism (PJ) is characterized by conscious attitude of journalists and media. Is proposed like an informative form that intend to approach to the daily existence of the reader. In this context, we study how this perspective -in detail Warning Call to the Public Administration- affects the institutional communication. The Public Administration becomes a receptor of media messages, with the aim of Public Administration acting in risk situations. Methodologically, this study presents a content analysis of more than 30,000 informative units reviewed on 406 samples from 12 diaries daily published. Also, we used a statistical analysis Biplot HJ.
\end{abstract}

Keywords

Communication, institutional communication, Journalism, Prevention Jounalism.

\section{INTRODUCCIÓN Y ESTADO DE LA CUESTIÓN}

El estudio de la comunicación de las organizaciones presenta una dilatada trayectoria en la investigación científica. En este sentido, parece necesario abordar esta comunicación -conocida como organizacional, corporativa (Capriotti, 1999) o institucional (Saladrigas, 2005)- desde

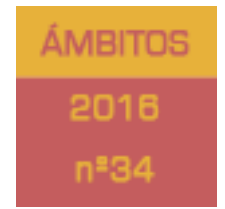
ópticas diferentes, como es el caso del Periodismo de Prevención (PdP). De hecho, para distintos estudiosos "uno de los ámbitos de más reciente desarrollo en la investigación sobre comunicación organizacional es el de la comunicación política” (Rodríguez Andrés, 2016). Se trata, el PdP, de una perspectiva periodística estudiada recientemente en España y que sugiere un enfoque basado en la gestión anticipada del 
riesgo en el entorno más próximo al ciudadano. Esta modalidad se define como aquella práctica periodística que, a través de una actitud consciente por parte de los periodistas y los medios, busca aproximarse a la cotidianeidad del lector a través de la publicación de información de carácter útil que permita una adecuada gestión de riesgos en su entorno próximo (Alende, 2015).

Este análisis se lleva a cabo en un momento en el que los medios de comunicación están inmersos en "procesos de transformación que implican un giro radical en la obtención y producción de contenidos, y en su relación con la audiencia" (Franklin, 2012). En esta encrucijada, explican Palomo y Palau-Sampio, "la innovación se presenta como un factor clave para afrontar la renovación estructural en un entorno presidido por la incertidumbre tecnológica y económica" (2016), tal y como refrendan Van-der-Haak, Parks y Castells (2012), Pavlik (2013) o Campos-Freire (2015). De hecho, en el estudio del periodismo contemporáneo se acepta ya que sus cambios "provocan una necesaria revisión de las diversas características de la profesión, desde los procesos de construcción de la opinión pública por parte del lector/usuario hasta la configuración de las redacciones y las funciones que en ellas se definen" (Renó y Renó, 2015).

El Periodismo de Prevención tiene, por tanto, su eje central precisamente en las personas. En el uso que estas pueden hacer de la comunicación periodística para tratar de evitar ciertas situaciones de riesgo y en las propias capacidades de esta corriente para cumplir esta demanda. En este sentido, puede adscribirse también a la línea de efectos de la comunicación sobre la conducta de los individuos (McQuail y Windahl, 1989: 98), pues en este contexto, "las sociedades modernas desarrollan un típico modelo [...] que provoca riesgos, contingencia y peligro" (Alfie y Méndez, 2000: 174). Es la llamada sociedad del riesgo (Beck, 1998: 14). Coincide, además, con la fase del periodismo contemporáneo (Guillamet, 2003: 43), en la que buena parte del imaginario social se funda en representaciones ofrecidas por una esfera mediática (Calle, 2005: 179) afectada por procesos de convergencia digital (Navarro, 2009).

Respecto a la influencia del Periodismo de Prevención en la comunicación de una organización, se acepta -en consonancia con autores como La Porte (2012) o Cancelo y Gadea (2013)- que la dimensión comunicativa de un organismo se asocia al precepto de su propia legitimación. Por tanto, el desarrollo comunicativo de un ente repercute directamente en dos aspectos fundamentales de su existencia: la reputación y la autoridad (Vázquez, 2015). Respecto a su evolución, Pasquel, Báez, Pauker y Apolo (2016) sostienen que las instituciones con el transcurso del tiempo han visto necesario fundamentar sus estrategias en comunicar una imagen comprensible, de confianza y credibilidad. Así, en este trabajo se sitúa a la Administración Pública, que generalmente es una de las fuentes de los medios de comunicación, como destinataria de unos mensajes, enviados por los medios.

El objetivo es el de realizar un Periodismo de Prevención para que la Administración actúe en determinados campos en un contexto, el actual, en el que "la comunicación debe superar un modelo de comunicación unidireccional y lineal, para dar lugar a procesos de relaciones públicas basados en la retroalimentación, en la que los individuos tengan cada vez un mayor protagonismo" (Baamonde, Pena y Martínez, 2015). Y para ello, de debe aclarar qué se entiende por institución. Como dice Sotelo (2001: 9) la idea de institución, que incluye a toda persona jurídica presente en la vida social, entiende la organización como una entidad radicalmente humana. Y concreta: es una organización humana en la que varias personas, a partir de unos principios configuradores y bajo una estructura concreta, coordinan diversas actividades para lograr determinados fines en el ámbito de una entidad social superior a la que sirve y proporciona cohesión y paradigma (2001: 24).

Interesa por tanto en esta introducción aclarar la naturaleza de esta comunicación en un momento en que "la aplicación de las tecnologías de la información posibilita que las capacidades comunicativas mejoren en eficacia y eficiencia debido a una especialización de las herramientas" (Alonso, 2016) y, por tanto, se modifica "el valor y la función de los contenidos" (Mateo, García y Guzmán, 2005). Al respecto, tras una investigación en la que se han establecido las bases teóricas del Periodismo de Prevención y se ha testado su presencia y características, se comprueba que la denominada "Llamada de atención a la Administración" puede entenderse como una fase previa del PdP. Es decir, en este caso los medios de comunicación son transmisores de mensajes que, emitidos por colectivos o particulares, se dirigen a las distintas administraciones con el fin de provocar en estos organismos una acción encaminada a solucionar una problemática concreta. Se reproduce así un modelo comunicativo diferente al más habitual en los medios de masas, donde la institución como fuente frecuente de los periodistas se convierte ahora en destinataria del mensaje -en virtud de su carácter de autoridad 
competente en cada materia- que emiten otros agentes, puesto que los "objetivos de cambio social reclaman discursos construidos desde y hacia emociones movilizadoras y creencias en la posibilidad de cambio" (Nós Aldás \& Santolino, 2015).

Abordar la capacidad de prevención de los medios de comunicación implica, además, detenerse en las funciones asociadas a los mismos. Sobre estos quehaceres, existen distintas taxonomías estudiadas por autores como Wright (1972), Martínez Terrero (2006), José Luis Martínez Albertos (1972), Denis McQuail (1991 y 1998) o Emil Dovifat (1980). Algunas de estas acciones relativas a la labor de informar entroncan con el bien común y la acción en el entorno, pues el fin social de los medios de comunicación puede resumirse como la obligación de las empresas mediáticas de buscar, antes que su propia rentabilidad económica, el beneficio de la sociedad (Tallón,1992: 19). Una idea que refrendan otros autores como Maciá (2013: 853) o Coca y Peñalva (1998: 23-24). En esta labor emerge la capacidad de los medios de actuar en su propio entorno y permitir anticiparse a ciertas situaciones. Esta tarea orientadora de los medios fue esbozada ya por Wright (1972: 19) y, en los años setenta del pasado siglo, "la sociedad experimentó un giro de tal naturaleza que el énfasis de las noticias empieza a recaer en las preocupaciones sociales" (Diezhandino, 1994: 24).

A lo largo del siglo XX fueron apareciendo entonces nuevas formas de informar como un modo de dar respuesta a distintas demandas (López, 2012: 23). Son concepciones diferentes del periodismo entre las que Xosé López destaca la Investigación Periodística, el Periodismo de Servicio, el Periodismo Estratégico o el Periodismo Preventivo(Bernabé, 2007). Cabe señalar que aquí se ha optado por la denominación Periodismo de Prevención frente al Periodismo Preventivo, porque a diferencia de este último -centrado en grandes crisis y conflictos en el ámbito internacional-, el PdP extiende su punto de mira a cualquier territorio, por pequeño que sea, en el que se perciban posibilidades de prevención desde la perspectiva mediática.

Entre las corrientes aceptadas como antecesora de este enfoque de prevención se encuentra el periodismo de/para la paz (López, 2012: 98). Pero se entienden también como otros antecedentes del PdP el periodismo cívico o público, nacido contra la apatía de la ciudadanía por las instituciones (Dader, 1999: 16); el periodismo de anticipación, estratégico o de soluciones -impulsado por Claude Monnier como un periodismo que permita al lector resolver problemas (1988)-; el Periodismo Premonitorio (Martínez Muñoz, 2002) o el Periodismo Social, que asume la responsabilidad del periodismo en los procesos sociales (Muñoz, 2011: 27). Cabe señalar que reflexionar sobre el Periodismo de Prevención en España exige acudir especialmente al trabajo realizado por el Instituto de Periodismo Preventivo y Análisis Internacional (Ippai), cuyas aportaciones están enfocadas al ámbito internacional (Bernabé, 2007). Así, en consonancia con la idea propuesta por Xosé López de que "otro periodismo es posible" y de que "solo queda, pues, intentar reflexionar cómo hacerlo y establecer las estrategias adecuadas" (2012: 12), para autores como Luhmann, Giddens o Beck, "las sociedades modernas desarrollan un típico modelo industrial y tecnológico que conduce a una serie de cursos de acción, de efectos que provocan riesgos" (Alfie y Méndez, 2000: 174). En consonancia con Piñuel, Gaitán y Lozano (2013), la práctica social del periodismo brinda al sujeto recursos y habilidades para desenvolverse en el medio social. Por tanto, entienden Piñuel, Gaitán y Lozano que un peligro se percibe cuando en el capital cognitivo del sujeto se dispone de discursos genéricos que categorizan los rasgos asociados a situaciones no suficientemente previstas (2013). Contribuye a ello el discurso periodístico (Gutiérrez Vidrio, 2010).

\section{MATERIAL Y MÉTODOS}

Tras la exposición de la conceptualización teórica en la que se asienta este trabajo, se presenta la investigación aplicada realizada para el estudio de la influencia del Periodismo de Prevención en la Comunicación Institucional. Este estudio nace del interés por estudiar cómo a través del Periodismo de Prevención -y, en concreto, a través de la fase denominada Llamada de Atención a la Administración- los medios de comunicación actúan como transmisores de mensajes que, emitidos por colectivos o particulares, se dirigen a las distintas administraciones con el fin de provocar en estos organismos una acción encaminada a solucionar una problemática concreta. La novedad de la temática aquí planteada se entiende como una de las principales características de esta investigación. Hasta el momento, no se han encontrado estudios similares. Así, se plantearon tres hipótesis, con la pretensión de su posterior corroboración. 
Hipótesis 1. En el contexto del Periodismo de Prevención, se reproduce un modelo comunicativo diferente al más habitual en los medios de masas, donde a través de la Llamada de Atención a la Administración, la institución como fuente frecuente de los periodistas se convierte en destinataria del mensaje que emiten otros agentes.

Hipótesis 2. La existencia y o cumplimiento de protocolos vinculados al bienestar público conforman, en el nivel de contenido, el principal tipo de mensaje de este modelo comunicativo.

Hipótesis 3. Temáticamente, la seguridad, los servicios públicos y el medio ambiente son los aspectos cuantitativamente más presentes en este modelo comunicativo.

El conjunto de esta hipótesis hacen referencia a una fase concreta del PdP, la mencionada Llamada de Atención a la Administración. En este estudio, las distintas unidades analizadas se sometieron a un proceso de clasificación en las siguientes fases:

1. Periodismo de Prevención-Óptimo: piezas informativas que cumplían con claridad alguno de los criterios de inclusión.

2. Periodismo de Prevención en fase de alerta: piezas informativas que, aun cumpliendo alguno de estos criterios, no ofrecían claramente datos de prevención pero sí constituyen una alerta.

3. Llamada de atención a la Administración: piezas informativas que, pese a no ser constituyentes de acciones preventivas en sí mismas, sí podían servir como medio para que la Administración competente en una determinada materia actuase a favor de la prevención.

4. Antesala de la prevención: piezas informativas que se aproximaban a alguna de las situaciones descritas en los criterios preventivos pero sin llegar a cumplir en su totalidad alguno de ellos.

5. Prevención implícita: piezas informativas que, sin ofrecer claramente contenidos preventivos, sí contenían algunos datos para que el lector pudiese anticiparse.

Se estudiaron un total de 12 diarios generalistas y de pago españoles: El Correo Gallego, La Región, El Progreso, Diario de Pontevedra, Diario de Arousa, Diario de Ferrol, El Ideal Gallego, Diario de Bergantiños, Atlántico Diario, La Opinión de A Coruña, La Voz de Galicia y Faro de Vigo. En estos dos últimos casos, se incluyeron en la muestra de análisis los ejemplares correspondientes a cada una de las ediciones de ambas cabeceras: La Voz de Galicia (A Coruña, A Mariña, Arousa, Barbanza, Carballo, Deza, Ferrol, Lemos, Lugo, Ourense, Pontevedra, Santiago y Vigo)y Faro de Vigo (Arousa, Deza, O Morrazo, Pontevedra, Ourense y Vigo). La muestra suma, en conjunto, 29 ediciones diferentes por cada día de estudio. Estos periódicos fueron revisados durante 14 meses.

Se recuperaron, en concreto, los ejemplares correspondientes a las siguientes fechas: 14 de noviembre de 2012, 15 de noviembre de 2012, 15 de diciembre de 2012, 15 de enero de 2013, 15 de febrero de 2013, 15 de marzo de 2013, 15 de abril de 2013, 15 de mayo de 2013, 15 de junio de 2013, 15 de julio de 2013, 15 de agosto de 2013, 15 de septiembre de 2013, 15 de octubre de 2013, 15 de diciembre de 2013. Cabe matizar que la primera intención de esta investigación consistía en analizar cada uno de los diarios publicados en Galicia el día 15 de cada mes durante el año 2013. Con esta determinación, se pretendía, partiendo de una muestra aleatoria, contar con un volumen de material lo suficientemente representativo del fenómeno objeto de estudio.

Según estos parámetros, esa primera selección quedaba conformada del siguiente modo: dos lunes (15 abril y 15 de julio), dos martes (15 de enero y 15 de octubre), un miércoles (15 de mayo), un jueves (15 de agosto), tres viernes (15 de febrero, 15 de marzo y 15 de noviembre), un sábado (15 de junio) y dos domingos (15 de septiembre y 15 de diciembre). Con el objetivo de obtener la misma representatividad por cada día de la semana se decidió ampliar esta muestra a 14 días, sumados dos de los meses de noviembre y diciembre de 2012. De este modo, se eliminó un viernes de 2013 (había 3) y se optó por introducir un miércoles, un jueves y un sábado más de la tercera semana de noviembre y diciembre de 2012. En concreto, se eligieron el miércoles 14 de noviembre de 2012 (es el más cercano al 15), el jueves 15 de noviembre de 2012 y el sábado 15 de 
diciembre de 2012. A nivel temporal, la muestra final quedó conformada, entonces, del siguiente modo: dos lunes ( 15 abril y 15 de julio de 2013), dos martes (15 de enero y 15 de octubre de 2013), dos miércoles (15 de mayo de 2013 y 14 de noviembre de 2012), dos jueves (15 de agosto de 2013 y 15 de noviembre de 2012), dos viernes (15 de febrero y 15 de marzo de 2013, pues se eliminó el 15 de noviembre, por contar con mayor representación de este mes correspondiente a 2012), dos sábados (15 de junio de 2013 y 15 de diciembre de 2012) y dos domingos (15 de septiembre y 15 de diciembre de 2013).

Por, tanto, se analizaron 406 ejemplares (29 ediciones por 14 días) y más de 30.000 unidades informativas. Una vez se determinó el material de análisis, se inició la fase de identificación de aquellas unidades informativas que podían vincularse a la tendencia Periodismo de Prevención y de la catalogación de estas unidades resultó la muestra de estudio definitiva a la que se aplicó el análisis de contenido propuesto y un estudio cualitativo de los mensajes emitidos. A la hora de discernir, en cada uno de los periódicos analizados, si una información podía considerarse o no como PdP, se tomaron en cuenta los siguientes criterios de identificación concretos, derivados de la revisión y el planteamiento teórico realizados para el PdP:

A. Se derivaron de una intención evidente del periodista por tomar posición respecto al bienestar del público.

B. Incidieron desde una perspectiva de vigilancia o control, en elementos de riesgo.

C. Valoraron la existencia de protocolos y su idoneidad.

D. Llamaron la atención sobre asuntos que solían causar situaciones de riesgo.

E. En el caso de derivarse de un suceso reciente, no se redujeron al mero relato de esos hechos sino que avanzaban en su comprensión proponiendo soluciones útiles.

F. Guías: ofrecieron indicaciones.

G. Buscaron, de manera clara e inequívoca, anticipar, avisar, alertar o posicionar al lector.

Una vez aplicados estos criterios a la revisión íntegra de los 406 ejemplares seleccionados, se logró identificar un total de 150 unidades informativas que respondían a criterios de Periodismo de Prevención. Su estudio en detalle se llevó a cabo mediante un análisis de contenido para el que se utilizó como instrumento de codificación un code-book compuesto por las siguientes variables: fase, fuentes, destinatarios, criterios y temática. A la hora de abordar este problema de investigación, se optó por el análisis de contenido como metodología de estudio porque se trata de un enfoque de investigación "específico" y "empleado con frecuencia en todas las áreas relacionadas con los medios" (Wimmer y Dominick, 1996: 169). Krippendorf, por su parte, lo define como "una técnica de investigación capaz de realizar inferencias válidas y estables (replicables) a partir de unos datos, en torno a su contexto" (Wimmer y Dominick, 1996: 170). Al respecto, Urchaga (2009) apunta que se trata de una técnica científica, objetiva y sistemática, que estudia cuantitativamente material cualitativo no estructurado o libre.

\section{ANÁLISIS Y RESULTADOS}

A continuación, se ofrecen los resultados derivados de esta investigación, unos datos que se exponen atendiendo al orden de hipótesis planteado en la metodología.

Hipótesis 1. En el contexto del Periodismo de Prevención, se reproduce un modelo comunicativo diferente al más habitual en los medios de masas, donde a través de la llamada de atención a la Administración, la institución como fuente frecuente de los periodistas se convierte en destinataria del mensaje que emiten otros agentes.

Para valorar con pertinencia la presencia de la fase de PdP identificada como Llamada de Atención a la Administración interesó en primer lugar conocer la representatividad de esta perspectiva en términos generales. Así, en el total de cabeceras analizadas fueron contabilizadas 31.545. Estos datos mostraron que el número de 
unidades de PdP halladas es muy bajo (150) ya se compare con el total de unidades (31.545) o con el número de ejemplares (406).

Pero además de cuantificar la presencia global de esta perspectiva en la prensa, el estudio diferenciador de fases realizado permite conocer datos relativos a las distintas etapas de prevención. Así, respecto a las fases se pudo observar que de estas 150 unidades de PdP analizadas, 64 (un 42,7\%) se identificaban claramente con PdP en fase óptima (unidades informativas que cumplían con claridad algunos de los criterios de inclusión). Sin embargo, otras 86 (un $57,3 \%$ ) se aproximaban de alguna manera a este nivel pero todavía en una fase previa. Es el caso de 47 unidades correspondientes a llamadas de atención a la Administración, de 19 que ofrecían datos de prevención de manera implícita, de otras 15 que constituían una alerta y de 5 identificadas como antesala de la prevención. Por lo tanto, los contenidos correspondientes a la categoría Llamada de Atención a la Administración -en la que se centra este estudio- suponen, cuantitativamente, la principal fase previa del Periodismo de Prevención. Las 47 unidades identificadas como correspondientes a esta fase superan ampliamente las 19 de la fase previa cuantitativamente más cercana, la Implícita. Por otra parte, la Llamada de Atención a la Administración presenta tan solo 17 unidades menos que la fase de PdP Óptimo, razones por las que resulta fundamental abordar en detalle el estudio de esta fase con intención de ahondar en el conocimiento sobre Periodismo de Prevención.

Para ello, resultó fundamental proceder al estudio de los agentes que intervienen como emisores y receptores del mensaje de prevención en esta fase concreta, puesto que en el contexto actual "los ciudadanos tienen cada vez más posibilidades para acceder al conocimiento, pero también van perdiendo la capacidad para seleccionar lo que más puede interesarles y mejor les puede informar" (De Ramón Carrión, 2015).

En relación a los destinatarios, no se observaron diferencias claras entre fases en el caso de la sociedad en general, siendo este el principal colectivo en casi todas las fases (97,3\% sobre el total). El PdP Óptimo tuvo como segundo colectivo el particular $(31,7 \%)$, situándose este porcentaje por encima de la media (18,7\%). En el PdP Óptimo hubo siete casos dirigidos a un organismo y no se encontraron unidades destinadas a empresas $u$ otras entidades. Sin embargo, en la fase Llamada a la Administración sobresalió el destinatario Organismo $(57,4 \%)$, una categoría identificada con las distintas administraciones públicas.

Como puede observarse, esta fase destaca por presentar este destinatario en mayor medida que el resto y permite conocer cómo en una amplia mayoría de los mensajes incluidos en esta fase los medios de comunicación son transmisores de mensajes que, emitidos por colectivos o particulares, se dirigen a las distintas administraciones con el fin de provocar en estos organismos una acción encaminada a solucionar una problemática concreta, tal y como demostró el análisis cualitativo de estos mensajes. Se reproduce así, como se explicaba, un modelo comunicativo diferente al más habitual en prensa y en el que las administraciones suelen ocupar el papel de fuente, entendidas como "los actores que los periodistas observan o entrevistan, incluidos los entrevistados que hablan por los medios o que son citados en artículos de revista, y también los que solo suministran información de antecedentes o sugieren temas y narraciones" (Gans, 2004). Así lo constata Rodrigo Alsina, que sostiene que fuentes muy presentes son las político-institucionales. "Estas son fuentes que aparecen a través, predominantemente, de sus propios comunicados y que suelen tener un acceso directo a los medio" (2005). 
Destinatario

Fase

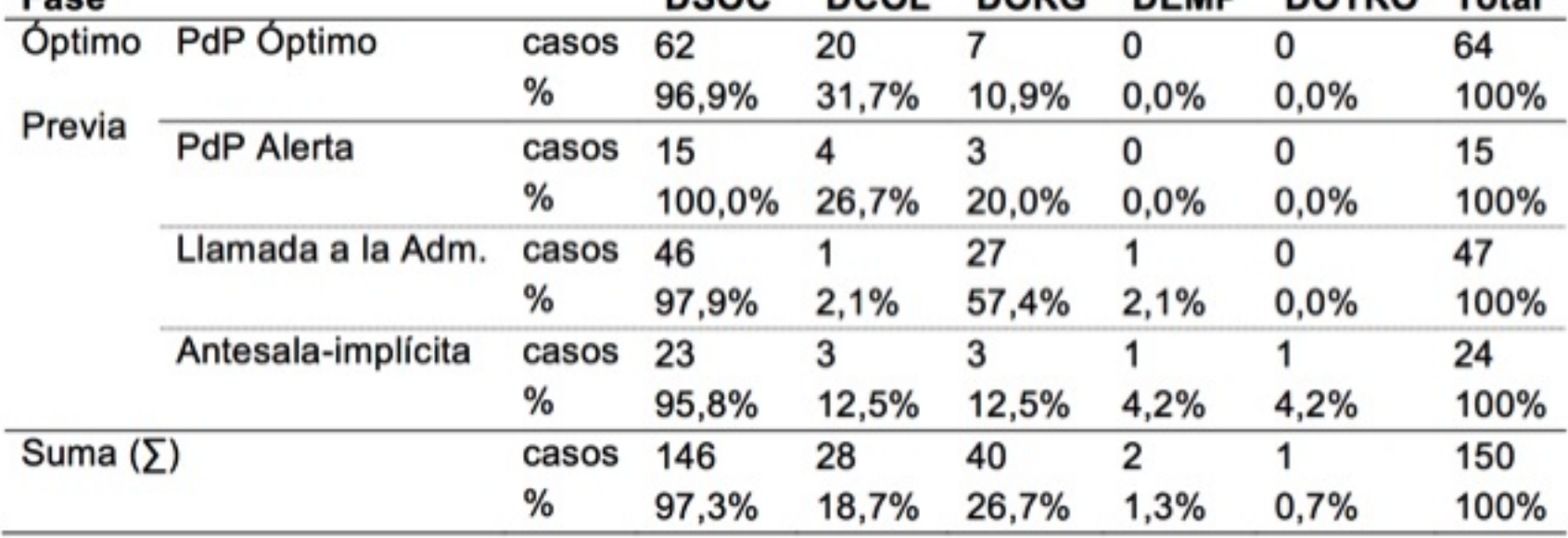

Gráfica 1. Contingencia fase-destinatario. Elaboración propia. Notación. DSOC (dirigido a la sociedad en general), DCOL (a un colectivo concreto), DORG (a un organismo oficial), DEMP (a una empresa) y DOTRO (a otro destinatario).

Con respecto al tipo de fuente, las distintas fases del PdP mostraron una tendencia general al empleo de fuentes expertas $(61,3 \%)$ y oficiales $(50 \%)$. En el caso del PdP Óptimo se citaron preferentemente dichas fuentes especializadas (Expertas: 81,3\%; Oficiales: 51,6\%), por lo que se puede afirmar que se utilizaron mejores fuentes en comparación con el resto de las fases. En su caso, el estudio de la Llamada de Atención a la Administración permite comprobar cómo si bien las fuentes particulares son las más presentes en relación al tipo de fuente, si se establece una comparativa de contingencia entre fases se observa que los informantes políticos tienen en esta fase una presencia mucho mayor que en las demás.

Fuentes

\begin{tabular}{|c|c|c|c|c|c|c|c|c|}
\hline \multirow{2}{*}{$\begin{array}{l}\text { Fase } \\
\text { Óptimo }\end{array}$} & & & FPOL & FEXPE & FPART & FOFIC & FOTRAS & Total \\
\hline & \multirow[t]{2}{*}{ PdP Óptimo } & Casos & 1 & 52 & 7 & 33 & 0 & 64 \\
\hline \multirow{7}{*}{ Previa } & & $\%$ & $1,6 \%$ & $81,3 \%$ & $10,9 \%$ & $51,6 \%$ & $0,0 \%$ & $100 \%$ \\
\hline & \multirow[t]{2}{*}{ PdP Alerta } & Casos & 1 & 9 & 5 & 7 & 0 & 15 \\
\hline & & $\%$ & $6,7 \%$ & $60,0 \%$ & $33,3 \%$ & $46,7 \%$ & $0,0 \%$ & $100 \%$ \\
\hline & \multirow[t]{2}{*}{ Llamada a la Adm. } & Casos & 7 & 15 & 30 & 21 & 1 & 47 \\
\hline & & $\%$ & $14,9 \%$ & $31,9 \%$ & $63,8 \%$ & $44,7 \%$ & $2,1 \%$ & $100 \%$ \\
\hline & \multirow[t]{2}{*}{ Antesala-implícita } & Casos & 2 & 16 & 10 & 14 & 0 & 24 \\
\hline & & $\%$ & $8,3 \%$ & $66,7 \%$ & $41,7 \%$ & $58,3 \%$ & $0,0 \%$ & $100 \%$ \\
\hline \multirow{2}{*}{\multicolumn{2}{|c|}{ Suma $(\Sigma)$}} & Casos & 11 & 92 & 52 & 75 & 1 & 150 \\
\hline & & $\%$ & $7,8 \%$ & 61,3 & $34.7 \%$ & $50 \%$ & $0,7 \%$ & $100 \%$ \\
\hline
\end{tabular}

Gráfica 2. Contingencia fase-fuentes. Elaboración propia. Notación. FPOL (fuentes políticas), FEXPE (fuentes expertas), FPART (fuentes particulares), FOFIC (fuentes oficiales) y FOTRAS (otras fuentes).

Por tanto, estos datos permiten afirmar que en el contexto del Periodismo de Prevención se reproduce un modelo comunicativo diferente al más habitual en los medios de masas, donde a través de la Llamada de Atención a la Administración, la institución como fuente frecuente de los periodistas se convierte en este campo también en destinataria del mensaje que emiten otros agentes. De esta manera, "a través de los medios de comunicación, también de la publicidad, se establece un nexo entre los emisores plurales que representan a los poderes sociales y económicos (el Estado, las corporaciones, los agentes económicos) y el público que 
construye y valida diariamente conceptos y autoconceptos sociales" (De Andrés del Campo y de Lima, 2014).

Hipótesis 2. La existencia y o cumplimiento de protocolos vinculados al bienestar público conforman, en el nivel de contenido, el principal tipo de mensaje de este modelo comunicativo.

Además de proceder al estudio de los agentes implicados en el mensaje de prevención, en esta investigación acerca de esta perspectiva periodística interesaba conocer también las razones que motivan la publicación de contenido perteneciente a la fase Llamada de Atención a la Administración. Para ello, se procedió al análisis de criterios de publicación.

En este punto, interesó conocer cuáles eran esos criterios o finalidades concretas. Así, por tipología, en el caso de la Llamada de Atención a la Administración, la existencia y/o cumplimiento de protocolos vinculados al bienestar público conforma el principal tipo de mensaje de este modelo comunicativo (CC).

A continuación, esta fase presenta además casos en los que se incidió en profundidad y desde una perspectiva de vigilancia o control, en elementos que -aun encontrándose en estado de calma- podían considerarse potenciales detonadores de circunstancias desagradables o negativas para el ciudadano (independientemente de su tipología: cuestiones de seguridad y protección, infraestructuras, procesos sociales...). También las que sacaban a la luz o llamaban la atención sobre cuestiones en las que, por su normalidad o cotidianeidad, no suele repararse pero que pueden constituir causas potenciales de riesgo (CB).

Hipótesis 3. Temáticamente, la seguridad, los servicios públicos y el medio ambiente son los aspectos cuantitativamente más presentes en este modelo comunicativo.

Respecto a la relación de la temática con la fase de prevención en que se inserta cada contenido, una tabla de contingencia permitió conocer este aspecto. Por su parte, en relación a la temática las unidades de la fase Llamada a la Administración presentaron como temas más recurrentes Seguridad personal/laboral (13 unidades sobre 47 totales), Infraestructuras o Servicios Públicos (11 unidades) y medio ambiente ( 9 unidades). En este sentido, se observa cómo este tipo de cuestiones son aquellas en las que más frecuentemente distintos agentes optan por dirigir sus demandas en materia de riesgos hacia las administraciones implicadas.

\begin{tabular}{|c|c|c|c|c|c|c|c|}
\hline \multirow{2}{*}{\multicolumn{2}{|c|}{ Temática }} & \multirow{2}{*}{\multicolumn{5}{|c|}{ Fase }} & Total \\
\hline & & & & & & & \\
\hline Agroganadera & casos & $\begin{array}{l}\text { PdP } \\
\text { Optimo } \\
0\end{array}$ & $\begin{array}{l}\text { Alerta } \\
1\end{array}$ & $\begin{array}{l}\text { Adm. } \\
2\end{array}$ & $\begin{array}{l}\text { Antes. } \\
0\end{array}$ & $\begin{array}{l}\text { Impl. } \\
0\end{array}$ & 3 \\
\hline & $\%$ & $0,0 \%$ & $33,3 \%$ & $66,7 \%$ & $0,0 \%$ & $0,0 \%$ & \\
\hline \multirow[t]{2}{*}{ Marítima } & casos & 0 & 1 & 4 & 0 & 0 & 5 \\
\hline & $\%$ & $0,0 \%$ & $20,0 \%$ & $80,0 \%$ & $0,0 \%$ & $0,0 \%$ & \\
\hline \multirow[t]{2}{*}{ Medioambiental } & casos & 3 & 0 & 9 & 2 & 0 & 14 \\
\hline & $\%$ & $21,4 \%$ & $0,0 \%$ & $64,3 \%$ & $14,3 \%$ & $0,0 \%$ & \\
\hline \multirow[t]{2}{*}{ Salud } & casos & 36 & 6 & 3 & 2 & 8 & 55 \\
\hline & $\%$ & $65,5 \%$ & $10,9 \%$ & $5,5 \%$ & $3,6 \%$ & $14,5 \%$ & \\
\hline \multirow[t]{2}{*}{ Entorno doméstico/familiar } & casos & 4 & 0 & 0 & 0 & 2 & 6 \\
\hline & $\%$ & $66,7 \%$ & $0,0 \%$ & $0,0 \%$ & $0,0 \%$ & $33,3 \%$ & \\
\hline \multirow[t]{2}{*}{ Entorno físico/clima } & casos & 2 & 0 & 2 & 0 & 1 & 5 \\
\hline & $\%$ & $40,0 \%$ & $0,0 \%$ & $40,0 \%$ & $0,0 \%$ & $20,0 \%$ & \\
\hline \multirow[t]{2}{*}{$\begin{array}{l}\text { Infraestructuras/ } \\
\text { servicios públicos }\end{array}$} & casos & 5 & 3 & 11 & 1 & 2 & 22 \\
\hline & $\%$ & $22,7 \%$ & $13,6 \%$ & $50,0 \%$ & $4,5 \%$ & $9,1 \%$ & \\
\hline \multirow[t]{2}{*}{ Económica/financiera } & casos & 1 & 2 & 3 & 0 & 2 & 8 \\
\hline & $\%$ & $12,5 \%$ & $25,0 \%$ & $37,5 \%$ & $0,0 \%$ & $25,0 \%$ & \\
\hline \multirow[t]{2}{*}{ Seguridad personal/laboral } & casos & 13 & 2 & 13 & 0 & 4 & 32 \\
\hline & $\%$ & $40,6 \%$ & $6,3 \%$ & $40,6 \%$ & $0,0 \%$ & $12,5 \%$ & \\
\hline Suma $(\Sigma)$ & casos & 64 & 15 & 47 & 5 & 19 & 150 \\
\hline
\end{tabular}


Gráfica 3. Contingencia temática-fase. Elaboración propia.

En este punto, pareció interesante -como aportación metodológica- la aplicación de un procedimiento estadístico multivariante que permitiese representar simultáneamente sobre un gráfico las relaciones entre las variables estudiadas y la posición concreta de la fase Llamada de Atención a la Administración. Para ello, se realizó un HJ BIPLOT (Vicente Villardón, 2010). Se seleccionaron aquellas variables que en el estudio previo discriminaban más el óptimo del resto. Se trata de Sección (local y sociedad), Destinatarios (colectivo), Género (noticia y entrevista), Página (portada), Titular, Firma, Número de fuentes, Utilidad (grado III) y Número de criterios. Se partió de la siguiente matriz de datos:

El biplot obtenido demostró que las distintas fases de PdP quedaban claramente separadas en el espacio y mostró cómo se relacionaban las variables analizadas. Sus dos ejes absorbieron el $85 \%$ de la información de la matriz inicial de datos. El eje 1 (horizontal) estuvo muy relacionado con la utilidad, por lo tanto se pudo denominar como dimensión de Periodismo de Prevención. Sobre dicho eje, se vio claramente que esta corriente ocupaba un extremo, el de la fase de Alerta la mitad y las dos fases restantes las dos posiciones más a la derecha. El eje 2 estaba más relacionado con la visibilidad de la información. Así, cuanto más en la parte inferior eran más visibles (estaban más presentes en titulares y género noticia). Por lo tanto, el PdP de la fase Llamada de Atención a la Administración o Alerta estaba más presente en titulares que en el caso de la fase de AntesalaImplícita. Por tanto, este aporte metodológico refuerza la entidad, como fases, de cada una de las categorías previas del Periodismo de Prevención. En el caso de la fase que aquí ocupa, la Llamada de Atención a la Administración, este método arroja información complementaria al análisis de contenido realizado.

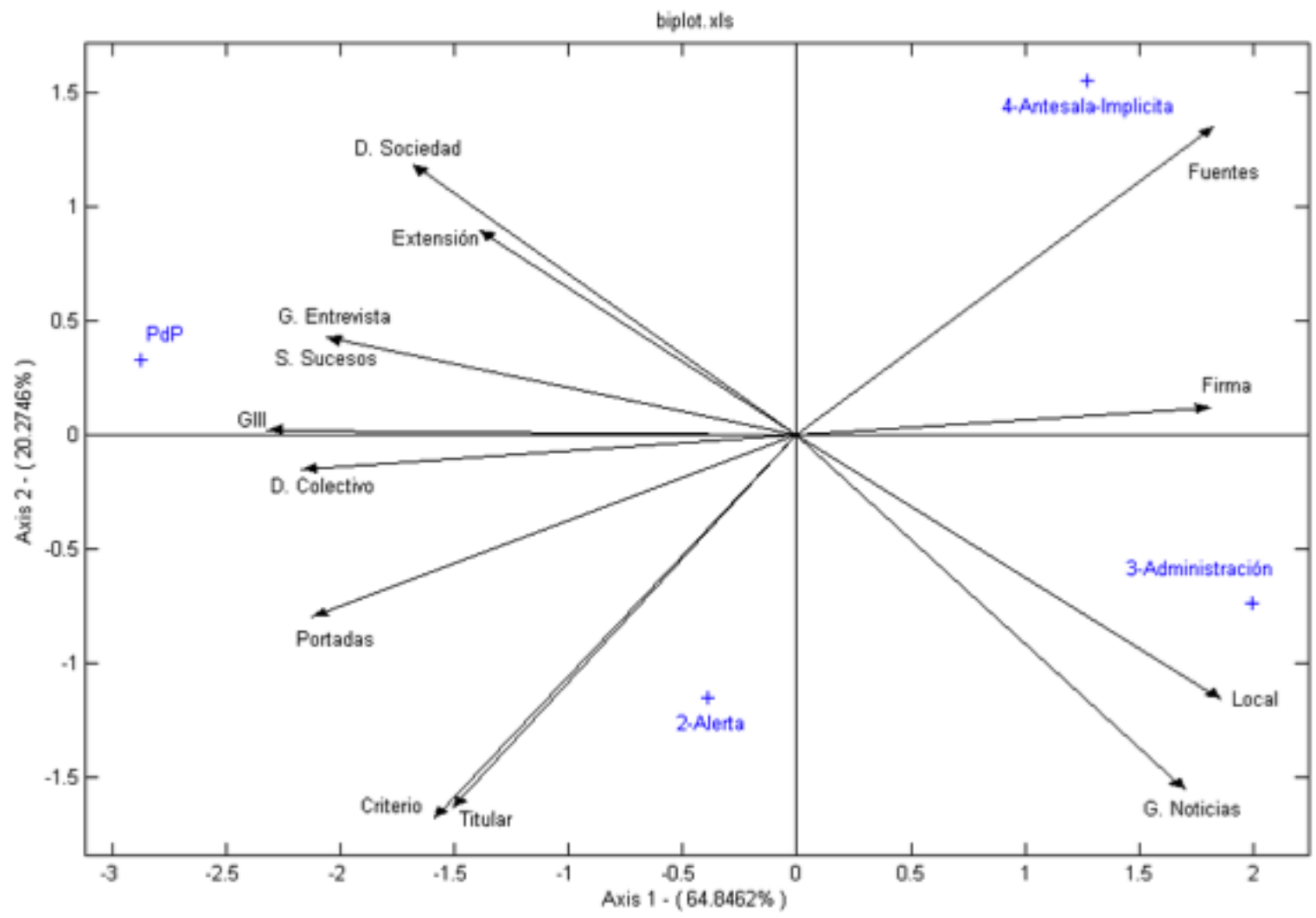

Gráfica 4. HJ Biplot. Elaboración propia.

\section{CONCLUSIONES}

Una vez realizado este análisis y habiendo comprobado la veracidad de las hipótesis planteadas, se puede concluir que:

La influencia del Periodismo de Prevención queda comprobada en la reproducción de un modelo comunicativo diferente al más habitual en los medios de masas, donde la institución como fuente frecuente de los periodistas 
se convierte ahora en destinataria del mensaje -en virtud de su carácter de autoridad competente en cada materia- que emiten otros agentes. A través de la difusión de estos mensajes, los medios dan voz a las demandas de colectivos o particulares destinadas a las distintas administraciones con la intención de que estas actúen en determinados campos de cada a la gestión de riesgos.

Asimismo, la importancia cuantitativa de este tipo de contenidos demuestra que la fase Llamada de Atención a la Administración supone la principal fase de Periodismo de Prevención. Por tanto, aunque no se trate todavía de una prevención en estado óptimo su existencia afecta a la comunicación institucional, que destaca por no ser únicamente la emitida por instituciones sino también la dirigida a las mismas con el fin de buscar solución a una problemática de riesgo. En el nivel temático, la seguridad y los servicios públicos figuran como los asuntos más frecuentes del temario de prevención en esta fase concreta. Se trata, por tanto, de un discurso mediático protagonizado por aspectos de competencia pública o administrativa.

\section{REFERENCIAS}

\subsection{Bibliográficas}

ALENDE, S. (2015): Periodismo de Prevención en Galicia. El concepto de comunicación útil en la prensa diaria . Tesis de doctorado, Universidade de Vigo. Disponible en http://www.investigo.biblioteca.uvigo.es/xmlui/handle/11093/350

ALONSO, M. (2016): "Comunicación empresarial de las empresas del IBEX 35 en Twitter”, Revista Mediterránea de Comunicación, 7(1), pp. 137-161.

doi: http://dx.doi.org/10.14198/MEDCOM2016.7.1.8

ALFIE, M. \& MENDEZ, L.H. (2000) : "La sociedad del riesgo: amenaza y promesa“, Sociológica, 43, pp. 173201.

BAAMONDE, X. M., PENA, A. \& MARTÍNEZ, X. (2015): "La gestión de la comunicación digital como herramienta de transparencia y rendición de cuentas en la ONGDs", Revista de la Asociación Española de Investigación de la Comunicación, 2(4), pp. 27-33.

ALSINA, M. R. (2005): La construcción de la noticia. Barcelona: Paidós.

BECK, U. (1998/Trad.): La sociedad del riesgo. Hacia una nueva Modernidad. Barcelona: Paidós.

BERNABÉ, J. (2007): Periodismo preventivo. Otra manera de informar sobre las crisis y los conflictos internacionales. Madrid: Los Libros de la Catarata.

CALLE, A. (2005): Nuevos movimientos globales. Hacia la radicalidad democrática. Madrid: Editorial Popular.

CAMPOS FREIRE, F.(2015): "Adaptación de los medios tradicionales a la innovación de los metamedios", El profesional de la información, v. 24, n. 4, pp. 441-450. doi: http://dx.doi.org/10.3145/epi.2015.jul.11

CANCELO, M. Y GADEA, G. (2013): "Empoderamiento de las redes sociales en las crisis institucionales", Revista de Comunicación Vivat Academia, 124, pp. 21-33. doi: http://dx.doi.org/10.15178/va.2013.124.21-33

CAPRIOTTI, P. (1999): “Comunicación corporativa. Una estrategia de éxito a corto plazo”, Reporte C\&DCapacitación y desarrollo, 13, pp. 30-33.

COCA, C. \& PEÑALVA, J.L. (1998): Modelos de los medios de comunicación social . Periodismo escrito. Zarautz: Servicio Editorial del País Vasco.

DE ANDRÉS, S. \& DE LIMA, R. (2014): "Análisis crítico del discurso publicitario institucional/comercial sobre las personas mayores en España [Critical Analysis of Government vs. Commercial Advertising Discourse on Older Persons in Spain]", Comunicar, 42, pp. 189-197. doi: http://dx.doi.org/10.3916/C42-2014-19 
DE RAMÓN CARRIÓN, M. (2015): "Análisis de los usos y prácticas profesionales. Datos sobre las redes en los medios de comunicación y en las empresas e instituciones", Estudios Sobre El Mensaje Periodístico, 21, pp. 4959. doi:10.5209/rev_ESMP.2015.v21.51134

DADER, J.L.: La recuperación ciudadana de los medios: vías de participación y contrapeso crítico de los consumidores y usuarios ante los medios de comunicación de masas. En VV.AA. (1999): Los usuarios de la sociedad de la información. Madrid: Ceaccu.

DOVIFAT, E. (1980): Política de la información. Navarra: Eunsa.

FRANKLIN, B. (2012): "The future of journalism: developments and debates", Journalism studies, v. 13, n. 5-6, pp. 663-681. doi: $h t t p: / / d x . d o i . o r g / 10.1080 / 1461670 X .2012 .712301$

GANS, H. (2004): Deciding what's news: a study of CBS eve- ning news, NBC nightly news, Newsweek and Time (25th anniversary edition). Evanston: Northwestern University Press.

GUTIÉRREZ VIDRIO, S. (2010): “Discurso periodístico: una propuesta analítica”, Comunicación y sociedad, 14, pp. 169-198.

GUILLAMET, J. (2003): "Por una historia comparada del periodismo. Factores de progreso y atraso", Doxa, 3, pp. 35-56.

LA PORTE, J.M. (2012): Introducción a la Comunicación Institucional de la Iglesia. Madrid: Palabra.

LÓPEZ, X. (2012): Movimientos periodísticos. Las múltiples iniciativas profesionales y ciudadanas para salvar los elementos básicos del periodismo en la era digital. Salamanca: Comunicación Social, Ediciones y Publicaciones.

MACIÁ, C. (2013): "Ética periodística, I+D+I y transferencia del conocimiento", Estudios sobre el Mensaje Periodístico, 19, pp. 853-862.

MARTÍNEZ MUÑOZ, Y. (2002): "El periodismo preventivo. El papel de los mass media en la prevención de conflictos armados", Estudios Internacionales de la Complutense, 4, pp. 149-171.

MATEO, J., GARCÍA, A., \& GUZMÁN, S. (2005): “El medio ya no es el mensaje”; Comunicar, 13 (25). doi: http://dx.doi.org/10.3916/25712

MCQUAIL, D. (1991) (2ª): Introducción a la teoría de la comunicación de masas. Barcelona: Paidós.

MCQUAIL, D (1998): La acción de los medios. Los medios de comunicación y el interés público. Buenos Aires: Amorrotu Editores.

MARTÍNEZ ALBERTOS, J.L. (1972): La información en una sociedad industrial. Madrid: Editorial Tecnos.

MARTÍNEZ TERRERO, J.M. (2006): Teorías de comunicación. Guayana: Universidad Católica.

MONNIER, C. (1988). "El nuevo periodismo de anticipación”, Nuestro Tiempo, 88, pp.50-59.

NAVARRO, L. (2009): "Tres lustros del periodismo digital: interactividad e hipertextualidad [Fifteen Years of Online Journalism. Interaction and Hypertextuality]", Comunicar, 33, pp. 35-43. doi: 10.3916/c33-2009-02-003.

NÓS ALDÁS, E. \& SANTOLINO, M. (2015): "La Investigación en Comunicación y Cooperación en los nuevos escenarios de movilización social: ONGD, objetivos de justicia social y eficacia cultural”, Revista de la Asociación Española de Investigación de la Comunicación, 2(4), pp. 1-7.

PALOMO, B., \& PALAU-SAMPIO, D. (2016): "El periodista adaptativo. Consultores y directores de innovación analizan las cualidades del profesional de la comunicación". El profesional de la información, 25(2), pp. 188-195.

PASQUEL, G., BÁEZ, V., PAUKER, L. \& APOLO, D. (2016): “Percepción, activos intangibles y stakeholders: 
modelo para el análisis de imagen corporativa", Revista Mediterránea de Comunicación, 7(1), pp. 179-196. doi: http://dx.doi.org/10.14198/MEDCOM2016.7.1.10

PAVLIK, J. V.(2013): "Innovation and the future of journalism", Digital journalism, v. 1, n. 2, pp. 181-193. doi: http://dx.doi.org/10.1080/21670811.2012.756666

PIÑUEL, J.L., GAITÁN J.A., Y LOZANO, C. (2013): Confiar en la prensa o no. Un método para el estudio de la construcción mediática de la realidad. Salamanca, Ed. Comunicación Social.

RENÓ, D., \& RENÓ, L. (2015): "Las nuevas redacciones, el "Big Data” y los medios sociales como fuentes de noticias”; Estudios Sobre El Mensaje Periodístico, 21, pp. 131-142. doi:10.5209/rev_ESMP.2015.v21.51135

RODRÍGUEZ ANDRÉS, R. (2016): "Retos de la comunicación política 2.0 en una sociedad que envejece", Revista Mediterránea de Comunicación, 7(1), pp. 1-14. doi: http://dx.doi.org/10.14198/MEDCOM2016.7.1.11

RODRIGO ALSINA, M. (2001): Teorías de la comunicación. Ámbitos, métodos y perspectivas. Barcelona: Ed. Universidad Autónoma de Barcelona.

SALADRIGAS, H. (2005): "Comunicación organizacional: Matrices teóricas y enfoques comunicativos", Revista Latina de comunicación social, (60), 8.

SOTELO, C. (2001): Introducción a la Comunicación Institucional. Ed. Ariel Comunicación. Barcelona

TALLÓN, J. (1992): Lecciones de empresa informativa. Madrid: Ediciones Ciencias Sociales.

URCHAGA, J.D.: Análisis de contenido. En REYES, R. (Coord.) (2009): Diccionario crítico de Ciencias Sociales. Madrid-México: Universidad Complutense \& Plaza-Valdés.

VAN-DER-HAAK, B.; PARKS, M.; CASTELLS, M.(2012): "The future of journalism: Networked journalism”, International journal of communication, v. 6, pp. 2913-2938.

VAZQUEZ, P. (2015) : "Tuits institucionales: del servicio al ciudadano a la proclama política“, Cuadernos Artesanos de Comunicación, 90.

VICENTE VILLARDÓN, J.L. (2010): Multibiplot: A package for Multivariate Analysis using Biplots. Departamento de Estadística. Universidad de Salamanca.

WIMMER, R. \& DOMINICK, J.R. (1996): La investigación científica de los medios de comunicación. Una introducción a sus métodos. Barcelona: Bosch

WRIGHT, R. (1972). Comunicación de masas. Buenos Aires: Paidós.

\subsection{Otras referencias}

DIALNET [Recurso online: http://goo.gl/vLYF4 /]

CENTRO SUPERIOR DE INVESTIGACIONES CIENTÍFICAS [Recurso online: http://goo.gl/CqoJ1B] INSTITUTO NACIONAL DE ESTADÍSTICA (INE) [Recurso online: http://goo.gl/LcEA]

Software estadístico SPSS 18.0.

TESEO: BASE DE DATOS [Recurso online: https://goo.gl/dkxBG] 
Dra. Silvia Alende Castro es doctora por la Universidad de Vigo. Los nuevos modelos comunicativos, la opinión pública o el impacto de las tecnologías de la comunicación en la práctica periodística centran sus principales ámbitos de estudio. Profesionalmente, su trayectoria está vinculada al trabajo en prensa y el impulso de una agencia de marketing digital.

Dra. Mercedes Román Portas. Profesora titular de la Universidad de Vigo. Área: Periodismo. Doctora en periodismo por la Universidad de Navarra. Ha puesto en marcha y es la coordinadora del doctorado en Investigación en Comunicación. En la actualidad es coordinadora de prácticas externas del grado de Comunicación Audiovisual.

Ámbitos. Revista Internacional de Comunicación, n.34, edición de otoño, 2016.

Recibido: $12 / 08 / 2016$

Aprobado: 05/09/2016 